\title{
Inducing probability distributions on the set of value functions by Subjective Stochastic Ordinal Regression
}

\author{
Salvatore Corrente ${ }^{\mathrm{a}}$, Salvatore Greco ${ }^{\mathrm{a}, \mathrm{d}}$, Miłosz Kadziński ${ }^{\mathrm{b}}$, Roman Słowiński $^{\mathrm{b}, \mathrm{c}}$ \\ ${ }^{a}$ Department of Economics and Business, University of Catania, Corso Italia, 55, 95129 Catania, Italy \\ ${ }^{b}$ Institute of Computing Science, Poznań University of Technology, 60-965 Poznań, Poland \\ ${ }^{c}$ Systems Research Institute, Polish Academy of Sciences, 01-447 Warsaw, Poland \\ ${ }^{d}$ University of Portsmouth, Portsmouth Business School, Centre of Operations Research and Logistics (CORL), \\ Richmond Building, Portland Street, Portsmouth PO1 3DE, United Kingdom
}

\begin{abstract}
Ordinal regression methods of Multiple Criteria Decision Aiding (MCDA) take into account one, several, or all value functions compatible with the indirect preference information provided by the Decision Maker (DM). When dealing with multiple criteria ranking problems, typically, this information is a series of holistic and certain judgments having the form of pairwise comparisons of some reference alternatives, indicating that alternative $a$ is certainly either preferred to or indifferent with alternative b. In some decision situations, it might be useful, however, to additionally account for uncertain pairwise comparisons interpreted in the following way: although the preference of $a$ over $b$ is not certain, it is more credible than preference of $b$ over $a$. To handle certain and uncertain preference information, we propose a new approach that builds a probability distribution over the space of all value functions compatible with the DM's certain holistic judgments. This distribution is parametrized to reflect different credibility levels of the supplied preferences. A didactic example shows the applicability of the proposed approach.
\end{abstract}

\footnotetext{
Email addresses: salvatore.corrente@unict.it (Salvatore Corrente), salgreco@unict.it (Salvatore Greco), milosz.kadzinski@cs.put.poznan.pl (Miłosz Kadziński), roman.slowinski@cs.put.poznan.pl (Roman Słowiński )

[Post-print] Please cite as: Corrente Salvatore, Greco Salvatore, Kadzinski Milosz, Słowiński Roman, Inducing probability distributions on the set of value functions by Subjective Stochastic Ordinal Regression, Knowledge-Based Systems, 112, 26-36
} 
Keywords: Multiple Criteria Decision Aiding, Ordinal Regression, Stochastic Multiobjective Acceptability Analysis, Multi-Attribute Value Function, Uncertain Preference Information, Probability Distribution

\section{Introduction}

Procedures designed to support choice, ranking, or sorting decisions concerning a set of alternatives in the presence of conflicting points of view, technically called criteria, is the domain of Multiple Criteria Decision Aiding (MCDA) (for a collection of state-of-the-art surveys, see [16]). We observe a growing demand for these procedures in various real-world decision problems, ranging from regional planning to finance. Within MCDA, three main approaches have been proposed:

- Multiple Attribute Value Theory [34], which assigns to each alternative a score representing its preferability;

- outranking approach $[6,17]$, which builds a crisp or fuzzy preference relation whose semantics is "alternative $a$ is at least as good as alternative $b$ ";

- decision rule approach [21,30], which represents preferences of the Decision Maker (DM) by means of "if ..., then ..." rules specifying conditions leading to some recommendation.

All these approaches require elicitation of preferences by the DM, in the form corresponding to the underlying preference model. From the viewpoint of preference elicitation, we can distinguish the following main types of preference model construction paradigms implemented in MCDA:

a) direct elicitation: the DM specifies directly values of parameters of the decision model at hand; for a long time, in many applications, this was the only way of fixing parameters of a decision model, e.g., weights of criteria used in a weighted sum model have been specified directly by the DM;

b) indirect elicitation based on Ordinal Regression (OR) [13, 25, 26, 42]: the DM supplies a set of decision examples, e.g., pairwise preference comparisons of some (real or fictitious) reference alternatives, and from this information a set of compatible preference model parameters is inferred; 
c) robust indirect elicitation based on Robust Ordinal Regression (ROR) [10, 11, 22]: the DM supplies, as in OR, a set of decision examples, but this information is used to construct the whole family of sets of compatible preference model parameters, which are all employed to build a recommendation for the DM; for an axiomatic characterization of the ROR, see [19];

d) stochastic indirect elicitation based on Stochastic Ordinal Regression (SOR) [31, 32]: the DM supplies a set of decision examples, as in OR and ROR, and all compatible sets of preference model parameters are considered as in ROR (see [2] for a recent contribution where ROR and SOR are applied to interacting criteria structured in a hierarchical way using the Choquet integral). However, in this case, not only the extreme cases of preference are considered, i.e., being true for all compatible sets of preference model parameters (necessary preference), or being true for at least one set of preference model parameters (possible preference), or holding for the most and the least advantageous sets of parameters (extreme ranks [29]); instead, a probability distribution $\mathcal{P}$ is considered over the family of all compatible sets of preference model parameters, and $\mathcal{P}$ is used to measure the part of this family for which alternative $a$ is preferred to alternative $b$, or the part for which $a$ is ranked in the $k$-th position.

In cases b)-d), the DM is asked to provide indirect preference information in which she compares with certainty some reference alternatives. Therefore, when she claims that $a$ is preferred to $b$ or that $a$ and $b$ are indifferent, she needs to be sure about the comparison of these two alternatives. However, in decision aiding processes, the DM may be uncertain about the comparison between two alternatives and, therefore, she may wish to express preferences with some degree of certainty or level of credibility. For example, she can say that the preference of $a$ over $b$ is more credible than the preference of $b$ over $a$, or that the preference of $a$ over $b$ is more credible than the preference of $c$ over $d$, admitting in this way that, on one hand, the preference of $a$ over $b$ is not certain and, on the other hand, that none of the two considered preferences is sure. For this reason, in general terms, we can distinguish between two types of preference information that could be provided by the DM:

- certain preference information, for which the DM has no doubt, such that all sets of compatible preference model parameters have to satisfy this type of preference information; 
- uncertain preference information, for which the DM has some doubt, such that not necessarily all sets of compatible preference model parameters have to satisfy this type of preference information.

The concepts of degree of preference, uncertain preference, or level of credibility are present in decision analysis in different contexts. The notion of graded preferences has been introduced in [37]. The DM can express her inclination to prefer one alternative over another, and one looks for a functional representation permitting a DM to express preferences of this type. For this reason, one needs to distinguish between a decisive DM, who is sure about the comparison of two alternatives, from an indecisive one, not being able to supply such a certain comparison.

In our perspective, the DM is decisive when she is able to provide certain preference information, and she is indecisive when she is willing to provide uncertain preference information. We allow our DM to express both certain and uncertain preferences. Note that the concepts of indecisiveness or incomplete preference have been also considered from an axiomatic point of view, e.g., in $[14,18]$.

As to the concept of uncertain or imprecise preference in MCDA, several contributions exist in the literature from which we highlight the following three. First, in contrast to the classical yes-no preference, the concept of fuzzy degree of preference has been introduced (for a survey, see [12], while for some representative papers considering fuzzy preferences in multiple criteria decision problems see $[4,15,33])$. Second, in [22], the authors allow the DM to express preferences that reflect different levels of credibility. Third, the concept of confidence level assigned to the decision examples provided by the DM is present in [38], and it is used within a procedure for identifying subsets of holistic judgments inconsistent with an assumed preference model.

In this paper, we propose a new approach that permits to take into account not only certain preference information that, is already considered in cases b)-d) discussed above, but also uncertain preference information provided by the DM. Starting from the certain information given by the DM, that should be always reproduced by the preference model at hand, we take into account the DM's uncertain preferences by constructing a probability distribution $\mathcal{P}$ over the space of models compatible with the certain preference information. Since the probability distribution $\mathcal{P}$ that we consider is based on the preference information supplied by the DM, we shall call the proposed 
procedure subjective stochastic indirect elicitation, and we will refer to the approach on which it is based as Subjective Stochastic Ordinal Regression (SSOR). SSOR has to be distinguished from the approach in which only certain preference information is taken into account and the probability distribution $\mathcal{P}$ is not based on the uncertain DM's preference information, that we shall call objective stochastic indirect elicitation, referring to the methodology on which it is based as Objective Stochastic

Ordinal Regression (OSOR). Without loss of generality, we consider a set of additive value functions as the DM's preference model.

We are convinced that consideration of the uncertain preference information makes the preference modeling more realistic and can be useful in any decision situation in which the DM wishes to express uncertain preferences. In this way, the DM is not forced to choose between strict preference and indifference, but she can also provide an intermediate level of preference. Moreover, we underline that the proposed approach is an extension of SOR since in case the DM is not willing to provide any uncertain preferences, it gives the same results as SOR.

The paper is organized as follows. In the next section, after recalling basic concepts of OR, ROR, and OSOR, we present the new SSOR approach, for the case of preference representation by means of additive value functions. In Section 3, we illustrate the use of SSOR by an application on a didactic example. Section 4 contains conclusions and proposals for future developments. All the proofs are provided in the Appendix.

\section{Subjective Stochastic Ordinal Regression (SSOR)}

Let us suppose that a set of alternatives $A=\{a, b, \ldots\}$ is evaluated on a set of criteria $G=$ $\left\{g_{1}, \ldots, g_{m}\right\}$. As a preference model, we consider a general monotonic additive value function:

$$
U(a)=u_{1}\left(g_{1}(a)\right)+\ldots+u_{m}\left(g_{m}(a)\right) \text { for any } a \in A \text {. }
$$

In the indirect preference elicitation mode, given a subset of reference alternatives $A^{R} \subseteq A$, relatively well known to the DM, she is asked to provide a pairwise preference relation $\succsim$ on $A^{R}(\succ$ and represent the asymmetric and the symmetric part of $\succsim$, respectively), where $a \succsim b$ iff $a$ is at least as good as $b$, and another preference relation $\succsim^{*}$ on $A^{R} \times A^{R}\left(\succ^{*}\right.$ and $\sim^{*}$ represent the asymmetric and 
the symmetric part of $\succsim^{*}$, respectively), where $(a, b) \succsim^{*}(c, d)$ iff the intensity of preference of $a$ over $b$ is at least as strong as the intensity of preference of $c$ over $d$, with $c \succsim d,\left(a, b, c, d \in A^{R}\right)$. This preference information can be considered as certain, in the sense that the DM has no doubt about the truth of supplied relations and, consequently, all value functions have to reproduce faithfully these relations.

To check if there exists at least one value function compatible with these preferences, one has to solve the following linear programming (LP) problem:

$$
\varepsilon_{\text {certain }}=\max \varepsilon, \text { subject to, }
$$

$$
\left.\begin{array}{l}
U(a) \geq U(b)+\varepsilon, \text { if } a \succ b, \text { for } a, b \in A^{R}, \\
\left.\begin{array}{l}
U(a)=U(b), \text { if } a \sim b, \text { for } a, b \in A^{R}, \\
U(a)-U(b) \geq U(c)-U(d)+\varepsilon, \\
U(c)-U(d) \geq \varepsilon, \\
\left.\begin{array}{l}
U(a)-U(b)=U(c)-U(d), \\
U(c)-U(d) \geq \varepsilon,
\end{array}\right\} \text { if }(a, b) \succ^{*}(c, d), \text { for } a, b, c, d \in A^{R}, \quad[c, d), \text { for } a, b, c, d \in A^{R}, \\
\sum_{j=1}^{m} u_{j}\left(x_{j}^{m_{j}}\right)=1, \quad u_{j}\left(x_{j}^{1}\right)=0, \text { for all } j=1, \ldots, m, \\
u_{j}\left(x_{j}^{k}\right) \leq u_{j}\left(x_{j}^{k+1}\right), \text { for all } k=1, \ldots, m_{j}-1, \text { and } j=1, \ldots, m,
\end{array}\right\} E_{\text {certain }}^{A^{R}} \\
{[N C]} \\
{[M C]}
\end{array}\right\}
$$

where $x_{j}^{k}, k=1, \ldots, m_{j}$, are all different values got by the alternatives on criterion $g_{j}$, with $x_{j}^{k}$ preferred to $x_{j}^{k-1}$ on criterion $g_{j}, k=2, \ldots, m_{j} ; x_{j}^{1}$ and $x_{j}^{m_{j}}$ are the worst and the best values an alternative can get on $g_{j},[\mathrm{NC}]$ and $[\mathrm{MC}]$ are normalization and monotonicity constraints, respectively, and $\varepsilon$ is an auxiliary variable used to transform the strict inequalities into weak inequalities. If $E_{\text {certain }}^{A^{R}}$ is feasible and $\varepsilon_{\text {certain }}>0$, then there exists at least one value function compatible with the preferences of the DM expressed on $A^{R}$.

While more than one value function could be compatible with the certain preference information provided by the DM, each of them can potentially give different recommendation for the non-reference alternatives in $A \backslash A^{R}$. In this regard, $\operatorname{ROR}[10,11,22]$ takes into account all value functions 
compatible with the preference information provided by the DM, building a necessary $\left(\succsim^{N}\right)$ and a possible $\left(\succsim^{P}\right)$ preference relation that hold for a pair of alternatives $(a, b) \in A \times A$ if $a$ is at least as good as $b$ for all or for at least one compatible value function, respectively. Given two alternatives $a$ and $b\left(a, b \in A \backslash A^{R}\right)$, it is very likely that $a$ is possibly preferred to $b$, and $b$ is possibly preferred to a but, generally, as observed in $[31,32]$, the share of compatible value functions for which $a$ is not worse than $b$ is different from the share of compatible value functions for which $b$ is not worse than $a$.

Remark that apart from the provided certain preference information, the DM could wish to express also uncertain preference information. For example, when comparing two alternatives $a$ and $b\left(a, b \in A^{R}\right)$, she may admit that $a$ could be preferred to $b$, and $b$ could be preferred to $a$ but, in her opinion, the preference of $a$ over $b$ is at least as credible as the preference of $b$ over $a$. She could also provide analogous preference information for quadruples of alternatives, stating that the preference of $a$ over $b$ is at least as credible as the preference of $c$ over $d\left(a, b, c, d \in A^{R}\right)$. We shall write $a \succsim_{L} b$ to indicate that the preference of $a$ over $b$ is at least as credible as the preference of $b$ over $a$; analogously, we shall write $(a, b) \succsim_{L}^{*}(c, d)$ to state that the preference of $a$ over $b$ is at least as credible as the preference of $c$ over $d$. To represent $\succsim_{L}$ and $\succsim_{L}^{*}$, we consider a probability distribution $\pi$ on the set $\mathcal{U}$ of value functions $U$ compatible with the certain preference information supplied by the DM, so that, for all $a, b, c, d \in A$

$$
a{ }_{L} b \Leftrightarrow \pi(\{U \in \mathcal{U}: U(a)>U(b)\}) \geq \pi(\{U \in \mathcal{U}: U(b)>U(a)\})
$$

and

$$
(a, b) \succsim_{L}^{*}(c, d) \Leftrightarrow \pi(\{U \in \mathcal{U}: U(a)>U(b)\}) \geq \pi(\{U \in \mathcal{U}: U(c)>U(d)\}) .
$$

In order to induce the probability $\pi$ from the preference information supplied by the DM, we consider the following discrete approximation of the above model, proceeding in the following two steps:

1. We sample $s_{v}$ value functions $U_{1}, U_{2}, \ldots, U_{s_{v}}$ in the set of compatible value functions $\mathcal{U}$, that is the set of value function satisfying monotonicity and normalization constraints, as well as constraints representing certain preference information provided by the DM. 
2. We assign a non-negative weight $w(U)$ to each value function $U$ sampled in the first step, such that $w\left(U_{1}\right)+\ldots+w\left(U_{s_{v}}\right)=1$, and the constraints representing the uncertain preference information are satisfied.

As to the first step, since the constraints representing the certain preference information provided by the DM, as well as the monotonicity and the normalization constraints define a convex set, the value functions can be sampled with the Hit-And-Run method (HAR; [43]) - its application in MCDA has been described in $[44,45]$.

The set of weights $w(U)$ assigned to the sampled compatible value functions can be seen as a probability distribution over the space of sampled functions, which permits to represent $\succsim_{L}$ and $\succsim_{L}^{*}$ as follows: for $a, b, c, d \in A^{R}$,

$$
a \succsim_{L} b \Leftrightarrow \sum_{t: U_{t}(a)>U_{t}(b)} w\left(U_{t}\right) \geq \sum_{t: U_{t}(b)>U_{t}(a)} w\left(U_{t}\right)
$$

and

$$
(a, b) \succsim_{L}^{*}(c, d) \Leftrightarrow \sum_{t: U_{t}(a)>U_{t}(b)} w\left(U_{t}\right) \geq \sum_{t: U_{t}(c)>U_{t}(d)} w\left(U_{t}\right) .
$$

Let us observe that the interpretation of the two preference relations $\succsim^{*}$ and $\succsim_{L}^{*}$ is completely different. On one hand, by saying that $(a, b) \succsim^{*}(c, d)$, the DM is sure about the preference of $a$ over $b$ and of $c$ over $d$, but the intensity of the first preference is not smaller than the intensity of the other one. On the other hand, when the DM states that $(a, b) \succsim_{L}^{*}(c, d)$, she is not certain on the preference of $a$ over $b$ and on the preference of $c$ over $d$, since it is also possible that for some value functions the two preferences are inverted. Here, the DM states only that the preference of $a$ over $b$ is at least as credible as the preference of $c$ over $d$.

Note 2.1. The uncertain preference information can be interpreted as in some well-known MCDA methods, such as AHP [41] and MACBETH [5]. Assume that the DM states that a is strongly preferred to $b, c$ is preferred to $d$, and e is weakly preferred to $f$. Then, this preference information can be represented by the following constraints:

$$
\sum_{t: U_{t}(a)>U_{t}(b)} w\left(U_{t}\right)>\sum_{t: U_{t}(c)>U_{t}(d)} w\left(U_{t}\right)>\sum_{t: U_{t}(e)>U_{t}(f)} w\left(U_{t}\right) .
$$

Indeed, the first inequality states that the preference of a over $b$ is more credible than the preference of $c$ over $d$, while the other inequality states that the preference of c over $d$ is more credible than the preference of e over $f$. 
From a computational point of view, to check if on the set of sampled valued functions there exists at least one probability distribution compatible with the uncertain preference information provided by the DM (in the following, for brevity, we shall write a compatible probability distribution to denote a vector of weights assigned to value functions sampled in the first step), one has to solve the following linear programming problem:

$$
\begin{aligned}
& \varepsilon_{L}^{*}=\max \varepsilon, \text { subject to } \\
& \sum_{t: U_{t}(a)>U_{t}(b)} w\left(U_{t}\right) \geq \sum_{t: U_{t}(b)>U_{t}(a)} w\left(U_{t}\right)+\varepsilon, \quad \text { if } a \succ_{L} b, \text { for } a, b \in A^{R}, \\
& \sum_{t: U_{t}(a)>U_{t}(b)} w\left(U_{t}\right) \geq \sum_{t: U_{t}(c)>U_{t}(d)} w\left(U_{t}\right)+\varepsilon, \quad \text { if }(a, b) \succ_{L}^{*}(c, d), \text { for } a, b, c, d \in A^{R} \\
& \sum_{t=1}^{s_{v}} w\left(U_{t}\right)=1 \\
& w\left(U_{t}\right) \geq 0, t=1, \ldots, s_{v} .
\end{aligned}
$$

If $E_{L}$ is feasible and $\varepsilon_{L}^{*}>0$, then there exists at least one probability distribution $\mathbf{w}$ compatible with the uncertain preference information provided by the DM.

Solving the optimization problem (2), one gets a single most discriminant probability distribution but, in general, there exists more than one compatible probability distribution. In order to take into account not only one but the whole set of compatible probability distributions, we propose to apply again the HAR method to extract a representative sample from the space defined by constraints $E_{L}$.

Using a sampled compatible probability distribution $\mathbf{w}$, the typical indices of the Stochastic Multiobjective Acceptability Analysis (SMAA) [35] can be computed:

- The rank acceptability index:

$$
R A I_{\mathbf{w}}(a, r)=\sum_{t: \operatorname{rank}\left(a, U_{t}\right)=r} w\left(U_{t}\right)
$$

where $\operatorname{rank}\left(a, U_{t}\right)$ denotes the position attained by alternative $a$ in the ranking constructed using the sampled value function $U_{t}$. It is the probability with which $a$ is ranked on the $r$-th position by this value function. 
- The pairwise winning index:

$$
P W I_{\mathbf{w}}(a, b)=\sum_{t: U_{t}(a)>U_{t}(b)} w\left(U_{t}\right)
$$

giving the probability that alternative $a$ is preferred to alternative $b$ for the sampled value function $U_{t}$.

For each sampled compatible probability distribution $\mathbf{w}$, the preference relation $\succsim_{L}^{\mathbf{w}}$, where $a \succsim_{L}^{\mathbf{w}} b$ means that $a$ is uncertainly preferred to $b$ with respect to distribution $\mathbf{w}$, can be defined as follows:

$$
a \succsim_{L}^{\mathbf{w}} b \Leftrightarrow P W I_{\mathbf{w}}(a, b) \geq P W I_{\mathbf{w}}(b, a) .
$$

Considering a sample of compatible probability distributions, as well as the totality of compatible probability distributions, one can define three different preference relations, in the set of alternatives $A$ for all $a, b \in A$ :

- $a \succsim_{L}^{R} b$ ( $a$ is uncertainly preferred to $b$ in a representative way) iff $P W I_{\mathbf{w}^{*}}(a, b) \geq P W I_{\mathbf{w}^{*}}(b, a)$, where $\mathbf{w}^{*}$ is the barycenter of all sampled compatible probability distributions obtained by averaging all of them, component by component; it can be considered as a representative compatible probability distribution;

- $a \succsim_{L}^{N} b$ ( $a$ is probabilistically necessarily preferred to $b$ ) iff the preference of $a$ over $b$ is at least as credible as the preference of $b$ over $a$, for all compatible probability distributions;

- $a \succsim_{L}^{P} b$ ( $a$ is probabilistically possibly preferred to $b$ ) iff the preference of $a$ over $b$ is at least as credible as the preference of $b$ over $a$ for at least one compatible probability distribution.

It is meaningful to observe that, while the relation $\succsim_{L}^{R}$ is based on a single compatible probability distribution, the preference relations $\succsim_{L}^{N}$ and $\succsim_{L}^{P}$ are obtained taking into account simultaneously the whole sample of value functions compatible with the certain preference information and all probability distributions on the sampled valued functions compatible with the uncertain preference information provided by the DM. From a computational point of view, given the two sets of constraints:

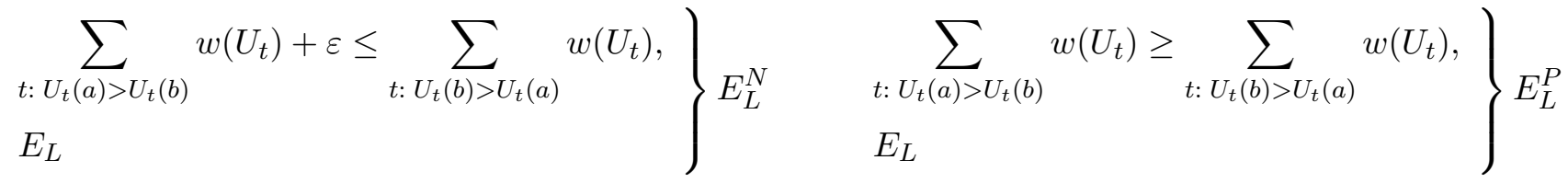


the probabilistic necessary and the probabilistic possible preference relations are computed as follows:

- $a \succsim_{L}^{N} b$ iff $E_{L}^{N}$ is infeasible or $\varepsilon_{L}^{N} \leq 0$, where $\varepsilon_{L}^{N}=\max \varepsilon$ subject to $E_{L}^{N}$;

- $a \succsim_{L}^{P} b$ iff $E_{L}^{P}$ is feasible and $\varepsilon_{L}^{P}>0$, where $\varepsilon_{L}^{P}=\max \varepsilon$ subject to $E_{L}^{P}$.

In the following proposition, we provide some important properties satisfied by the binary relations considered above, and defined taking into account the certain and the uncertain preference information provided by the DM.

\section{Proposition 2.1.}

1. For any compatible probability distribution $\mathbf{w}, \succsim_{L}^{\mathbf{w}}$ is strongly complete, that is, for all $a, b \in A$, $a \succsim_{L}^{\mathbf{w}} b$ or $b \succsim_{L}^{\mathbf{w}} a$

2. $\succsim^{N} \subseteq \succsim_{L}^{N} \subseteq \succsim_{L}^{R} \subseteq \succsim_{L}^{P} \subseteq \succsim^{P}$

3. For all $a, b \in A, a \succsim_{L}^{N} b$ or $b \succsim_{L}^{P} a$.

4. For all $a, b, c \in A$, if $a \succsim^{N} b$ and $b \succsim_{L}^{N} c$, then $a \succsim_{L}^{N} c$.

5. For all $a, b, c \in A$, if $a \succsim_{L}^{N} b$ and $b \succsim^{N} c$, then $a \succsim_{L}^{N} c$.

6. For all $a, b, c \in A$, if $a \succsim^{N} b$ and $b \succsim_{L}^{P} c$, then $a \succsim_{L}^{P} c$.

7. For all $a, b, c \in A$, if $a \succsim_{L}^{P} b$ and $b \succsim^{N} c$, then $a \succsim_{L}^{P} c$.

Proof. See Appendix.

Let us point out that if the DM is not able to provide any uncertain preference information, then our methodology boils down to SOR. Indeed, applying the HAR method to sample a certain number of probability distributions satisfying the non-negativity $[U N N C]$ and normalization constraints $[U N C]$ only, and computing their barycenter, is equivalent to considering a uniform probability distribution on the set of sampled value functions compatible with the certain preference information provided by the DM. For this reason, we claim that SSOR is a generalization of SOR.

Let us summarize all steps of the proposed methodology (see Figure 1): 
Figure 1: Flow chart of the proposed methodology

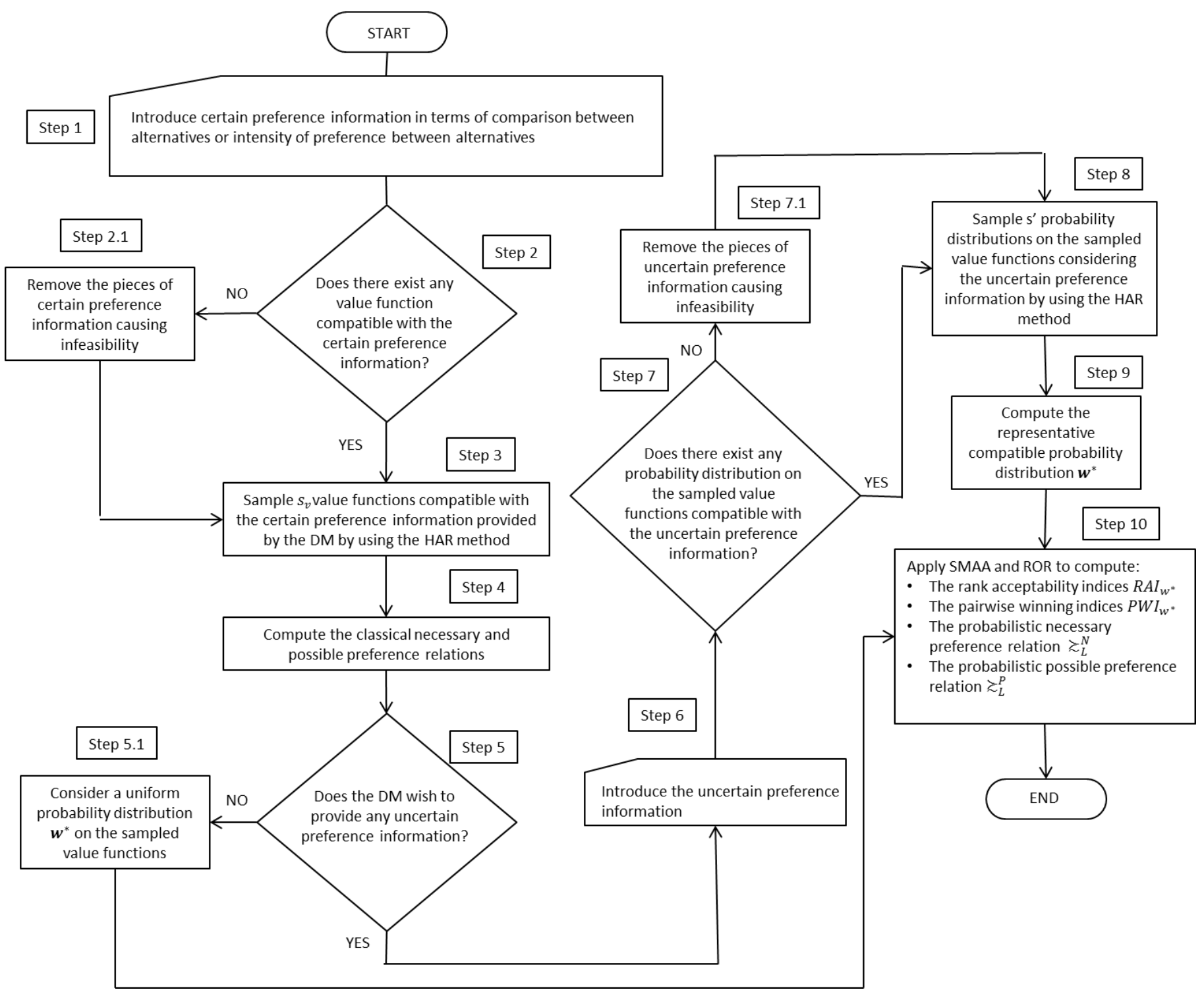

Step 1 The DM is asked to provide certain preference information in form of pairwise comparisons or statements concerning intensity of preference. Such preference information is translated to the constraints $[C P I C]$ in $E_{c e r t a i n}^{A^{R}}$.

Step 2 The analyst verifies whether there exists at least one value function compatible with the preference information provided by the DM by solving the LP problem (1). If so (i.e., if $\varepsilon_{\text {certain }}>0$ ), one can pass to Step 3). Otherwise, the pieces of certain preference information underlying the inconsistency have to be removed (Step 2.1) [39]. 
Step 3 Since more than one value function could be compatible with the certain preference information provided by the DM, we sample $s_{v}$ compatible value functions using the HAR method. The use of HAR is justified since the constraints $[C P I C]$ translating the DM's certain preference information combined with the monotonicity $[M C]$ and normalization $[N C]$ constraints define a convex polyhedron.

Step 4 In order to get robust recommendations with respect to the problem at hand, one can compute the "classical" necessary $\left(\succsim^{N}\right)$ and possible $\left(\succsim^{P}\right)$ preference relations taking into account the whole set of functions compatible with the certain preference information provided by the DM.

Step 5 The DM is asked whether she wishes to provide some uncertain preference information. If not, one can proceed to Step 5.1 where a uniform probability distribution on the sampled value functions is considered (in this case, the same weight is assigned to all sampled value functions), and then pass directly to Step 10. Otherwise, go to Step 6.

Step 6 The DM provides uncertain preference information expressing different degrees of credibility on the preference of one alternative over another. This preference information is translated to constraints $[U P I C]$ in $E_{L}$.

Step 7 The analyst verifies whether there exists some probability distribution on the value functions sampled in Step 3 that would be compatible with the DM's uncertain preference information by solving the LP problem (2). If so (i.e., if $\varepsilon_{L}^{*}>0$ ), then one can proceed to Step 8. Otherwise, the pieces of uncertain preference information underlying the incompatibility have to be removed (Step 7.1) [39].

Step 8 Since more than one probability distribution defined on the sampled value functions could be compatible with the uncertain preference information provided by the DM, we propose to sample $s^{\prime}$ of them by using the HAR method. Again, this is possible since constraints $[U P I C]$ translating this preference information combined with the non-negativity $[U N N C]$ and normalization $[U N C]$ constraints define a convex polyhedron. 
Step 9 A representative probability distribution $\mathbf{w}^{*}$ is computed by averaging component by component the probability distributions sampled in Step 8.

Step 10 The following robust recommendations for the problem at hand are derived:

- For each alternative $a$ and for each rank $r, r=1, \ldots,|A|$, the rank acceptability index $R A I_{\mathrm{w}^{*}}(a, r)$ is computed by considering the representative probability distribution $\mathbf{w}^{*}$;

- For each pair of alternatives $(a, b) \in A \times A$, the pairwise winning index $P W I_{\mathbf{w}^{*}}(a, b)$ is computed by considering the representative probability distribution $\mathbf{w}^{*}$;

- The truth of the probabilistic necessary and possible preference relations $\succsim_{L}^{N}$ and $\succsim_{L}^{P}$ is verified for all pairs of alternatives.

Let us emphasize that some modifications in the described methodology can be considered. For example, in Step 9, instead of the representative probability distribution $\mathbf{w}^{*}$ one can take into account another compatible probability distribution. Analogously, in Step 10, one can compute (as we shall show in the didactic example) the rank acceptability indices and the pairwise winning indices considering a uniform probability distribution on the sampled value functions.

\section{Illustrative example}

\subsection{Problem statement and results}

In this subsection, we illustrate the proposed approach using the example borrowed from [22]. The problem statement is the following. 15 sales managers are evaluated on three criteria: sales management experience $\left(g_{1}\right)$, international experience $\left(g_{2}\right)$, and human qualities $\left(g_{3}\right)$. Only 6 of the 15 managers are non-dominated and their performances are shown in Table 1. Let us suppose that the owner of the firm provided the following certain preference information (Step 1):

$$
\text { [Varlot } \left.\left.\succ \text { Petron] and [(Varlot, Petron }) \succ^{*} \text { (Ferret, Calvet }\right)\right] \text {. }
$$

After verifying that there exists at least one value function compatible with the DM's preferences (Step 2), we sampled 10,000 value functions satisfying the monotonicity $[\mathrm{MC}]$ and normalization $[\mathrm{NC}]$ constraints, as well as the two certain pieces of preference information provided by the owner 
Table 1: Performances of the sales managers on the three criteria.

$\begin{array}{rccc}\text { Sales manager } & g_{1} & g_{2} & g_{3} \\ \text { Bassama (B) } & 28 & 18 & 28 \\ \text { Calvet (C) } & 26 & 40 & 44 \\ \text { Ferret (F) } & 35 & 62 & 25 \\ \text { Frechet (R) } & 9 & 62 & 88 \\ \text { Petron (P) } & 6 & 15 & 100 \\ \text { Varlot (V) } & 62 & 43 & 0\end{array}$

(Step 3). Let us also suppose that the owner provides two pieces of uncertain preference information (Step 5):

$$
\text { [Calvet } \left.\succ_{L} \text { Frechet] and [(Varlot, Ferret }\right) \succ_{L}^{*} \text { (Calvet, Frechet)]. }
$$

By solving the LP problem (2), we get $\varepsilon_{L}^{*}>0$ (Step 6). This means that there exists at least one probability distribution on the set of sampled value functions restoring the uncertain preferences provided by the owner. After sampling 10,000 compatible probability distributions (Step 7), we computed the representative compatible probability distribution $\mathbf{w}^{*}$ (Step 8), and derived RAIs and $P W I$ s shown in Tables 2 and 3, respectively (Step 9).

In Table 2, we present both RAIs obtained for the representative probability distribution compatible with the DM's uncertain preferences, and for the uniform probability distribution on the set of sampled value functions (in brackets). Taking into account $R A I \mathrm{~s}$ for the first rank, and comparing the two types of results, we can observe that Varlot is the best among the six sales managers when considering the DM's uncertain preferences (first case), while Ferret is the best when considering only certain preference information under the hypothesis of a uniform probability distribution of the sampled value functions (second case). Moreover, while Ferret and Frechet are the second and the third in the first case, Varlot and Frechet are the second and the third in the other case. Observe also that Bassama and Petron are the worst sales managers in both cases.

Analogously to what has been observed for $R A I \mathrm{~s}$, some $P W I \mathrm{~s}$ in Table 3 do differ when considering the two sampling procedures. For example, given the uncertain preferences of the DM, when using the representative probability distribution, Varlot is preferred to Ferret with a probability of 
Table 2: Rank acceptability indices for the representative probability distribution w* compatible with DM's uncertain preferences. In brackets, there are rank acceptability indices for a uniform probability distribution on the sampled value functions.

\begin{tabular}{|c|c|c|c|c|c|c|}
\hline & $R A I_{\mathbf{w}^{*}}(a, 1)$ & $R A I_{\mathbf{w}^{*}}(a, 2)$ & $R A I_{\mathbf{w}^{*}}(a, 3)$ & $R A I_{\mathbf{w}^{*}}(a, 4)$ & $R A I_{\mathbf{w}^{*}}(a, 5)$ & $R A I_{\mathbf{w}^{*}}(a, 6)$ \\
\hline B & $0.0102 \quad(\mathbf{0 . 0 0 7 3})$ & $0.0189 \quad(\mathbf{0 . 0 1 9 7})$ & $0.1862(\mathbf{0 . 1 0 7 9})$ & $0.2137(\mathbf{0 . 1 9 1 5})$ & $0.4750 \quad(\mathbf{0 . 5 4 0 3})$ & $0.0960 \quad(\mathbf{0 . 1 3 3 3})$ \\
\hline C & $0.00(\mathbf{0 . 0 0})$ & $0.0152(\mathbf{0 . 0 2 5 0})$ & $0.3775 \quad(\mathbf{0 . 1 9 5 4})$ & $0.4717(\mathbf{0 . 5 5 8 0})$ & $0.1289 \quad(\mathbf{0 . 2 1 0 9})$ & $0.0066 \quad(\mathbf{0 . 0 1 0 7})$ \\
\hline $\mathrm{F}$ & $0.2695(\mathbf{0 . 4 5 1 3})$ & $0.6436(\mathbf{0 . 4 2 7 7})$ & $0.0829(\mathbf{0 . 1 1 3 9})$ & $0.0038(\mathbf{0 . 0 0 6 6})$ & $0.0002(\mathbf{0 . 0 0 0 5})$ & $0.00 \quad(\mathbf{0 . 0 0})$ \\
\hline $\mathrm{R}$ & $0.1587 \quad(\mathbf{0 . 2 6 7 5})$ & $0.1155 \quad(\mathbf{0 . 1 9 0 7})$ & $0.1974(\mathbf{0 . 3 3 2 2})$ & $0.2306 \quad(\mathbf{0 . 1 1 7 4})$ & $0.2827 \quad(\mathbf{0 . 0 8 9 4})$ & $0.0150 \quad(\mathbf{0 . 0 0 2 8})$ \\
\hline$P$ & $0.00(\mathbf{0 . 0 0})$ & $0.0027(\mathbf{0 . 0 0 0 9})$ & $0.0078(\mathbf{0 . 0 0 4 1})$ & $0.0156 \quad(\mathbf{0 . 0 1 8 9})$ & $0.0915 \quad(\mathbf{0 . 1 2 2 9})$ & $0.8823(\mathbf{0 . 8 5 3 2})$ \\
\hline $\mathrm{V}$ & $0.5616 \quad(\mathbf{0 . 2 7 3 9})$ & $0.2041 \quad(\mathbf{0 . 3 3 6 0})$ & $0.1482(\mathbf{0 . 2 4 6 5})$ & $0.0645(\mathbf{0 . 1 0 7 6})$ & $0.0215 \quad(\mathbf{0 . 0 3 6 0})$ & $0.00(\mathbf{0 . 0 0})$ \\
\hline
\end{tabular}

0.6042 while, when considering the certain preferences only and, therefore, a uniform probability distribution on the sampled value functions, Varlot is preferred to Ferret with a probability of only 0.3374. This means that, on average, Varlot is preferred to Ferret if we considered the uncertain preferences of the DM, while Ferret is preferred to Varlot if we considered certain preferences only.

Table 3: Pairwise winning indices for the representative probability distribution $\mathbf{w}^{*}$ compatible with DM's preferences. In brackets, there are pairwise winning indices for a uniform probability distribution on the sampled value functions.

\begin{tabular}{|c|c|c|c|c|c|c|c|c|c|c|c|c|}
\hline & & B & & $\mathrm{C}$ & & $\mathrm{F}$ & & $\mathrm{R}$ & & $\mathrm{P}$ & & V \\
\hline B & 0.00 & $(0.00)$ & 0.2949 & $(0.2600)$ & 0.0217 & $(0.0148)$ & 0.3300 & $(0.1558)$ & 0.8974 & $(0.8630)$ & 0.0434 & $(0.0628)$ \\
\hline $\mathrm{C}$ & 0.7051 & $(0.7341)$ & 0.00 & $(0.00)$ & 0.00 & $(0.00)$ & 0.5002 & $(0.1629)$ & 0.9725 & $(0.9694)$ & 0.0879 & $(0.1467)$ \\
\hline $\mathrm{F}$ & 0.9783 & $(0.9852)$ & 1.00 & $(1.00)$ & 0.00 & $(0.00)$ & 0.8085 & $(0.6789)$ & 0.9957 & $(0.9960)$ & 0.3958 & $(0.6626)$ \\
\hline $\mathrm{R}$ & 0.6700 & $(0.8442)$ & 0.4998 & $(0.8371)$ & 0.1915 & $(0.3211)$ & 0.00 & $(0.00)$ & 0.9773 & $(0.9950)$ & 0.2530 & $(0.4237)$ \\
\hline$P$ & 0.1026 & $(0.1370)$ & 0.0275 & $(0.0306)$ & 0.0043 & $(0.4000)$ & 0.0227 & $(0.00$ & 0.00 & $(0.00)$ & 0.00 & $(0.00)$ \\
\hline 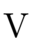 & 0.9566 & $(0.9372)$ & 0.9121 & $(0.8533)$ & 0.6042 & $(0.3374)$ & 0.7470 & $(0.5763)$ & 1.00 & $(1.00)$ & 0.00 & $(0.00)$ \\
\hline
\end{tabular}

In Tables 4(a) and 4(b), we present the probabilistic necessary and possible preference relations. For six pairs of sales managers, the probabilistic necessary preference relation holds; e.g., Ferret $\succsim_{L}^{N}$ Frechet. This means that the probability of the preference of Ferret over Frechet is not lower than the probability of the preference of Frechet over Ferret, independently of the sampled compatible probability distribution.

These results underline the usefulness of taking into account not only the certain preferences 
provided by the DM but also the uncertain ones. Indeed, considering the uncertain preferences brings to conclusions both on the ranking of the considered alternatives and on the pairwise comparisons between them, which are different from the conclusions drawn taking into account the certain preferences only.

Table 4: Probabilistic preference relations.

\begin{tabular}{cccccccccccccc} 
(a) Probabilistic necessary & \multicolumn{4}{c}{ (b) Probabilistic possible } \\
preference relation $\succsim_{L}^{N}$. & \multicolumn{4}{c}{ preference relation $\succsim_{L}^{P .}$} \\
B & 1 & 0 & 0 & 0 & 0 & 0 & $\mathrm{~B}$ & 1 & 1 & 1 & 1 & 1 & 1 \\
$\mathrm{C}$ & 0 & 1 & 0 & 1 & 0 & 0 & $\mathrm{C}$ & 1 & 1 & 0 & 1 & 1 & 0 \\
$\mathrm{~F}$ & 0 & 1 & 1 & 1 & 0 & 0 & $\mathrm{~F}$ & 1 & 1 & 1 & 1 & 1 & 0 \\
$\mathrm{R}$ & 0 & 0 & 0 & 1 & 0 & 0 & $\mathrm{R}$ & 1 & 0 & 0 & 1 & 1 & 1 \\
$\mathrm{P}$ & 0 & 0 & 0 & 0 & 1 & 0 & $\mathrm{P}$ & 1 & 1 & 1 & 1 & 1 & 0 \\
$\mathrm{~V}$ & 0 & 1 & 1 & 0 & 1 & 1 & $\mathrm{~V}$ & 1 & 1 & 1 & 1 & 1 & 1
\end{tabular}

\subsection{Discussion of results in view of properties of the preference relations}

In this section, we discuss in detail the results of our illustrative example in the context of Proposition 2.1. For clarity of presentation, we also show how the LP problems and the indices described in Section 2 are formulated and computed, respectively. Referring to the pairwise winning indices shown in Table 3 and remembering that $a \succsim_{L}^{*} b$ iff $P W I_{\mathbf{w}^{*}}(a, b) \geq P W I_{\mathbf{w}^{*}}(b, a)$ (see $\left.(3)\right)$, we can compute the preference relation $\succsim_{L}^{\mathbf{w}^{*}}$ shown in Table 5 .

1. For any compatible probability distribution $\mathbf{w}, \succsim_{L}^{\mathbf{w}}$ is strongly complete, that is, for all $a, b \in A$, $a \succsim_{L}^{\mathbf{w}} b$ or $b \succsim_{L}^{\mathbf{w}} a$

For each pair of sales managers $a, b \in A$, it is evident that $a \succsim_{L}^{\mathbf{w}^{*}} b$ or $b \succsim_{L}^{\mathbf{w}^{*}} a$ holds. For example, $C \succsim_{L}^{\mathbf{w}^{*}} B$ but $\operatorname{not}\left(B \succsim_{L}^{\mathbf{w}^{*}} C\right)$ or, analogously, $V \succsim_{L}^{\mathbf{w}^{*}} P$ but $\operatorname{not}\left(P \succsim_{L}^{\mathbf{w}^{*}} V\right)$. Let us observe that there is no pair of managers for which $a \succsim_{L}^{\mathbf{w}^{*}} b$ and $b \succsim_{L}^{\mathbf{w}^{*}} a$, i.e., there is no pair of managers for which the probability of $a$ is preferred to $b$ is the same as the probability of $b$ is preferred to $a$. 
Table 5: Preference relation $\succsim_{L}^{\mathbf{w}^{*}}$ computed considering the representative probability distribution $\mathbf{w}^{*}$ compatible with the uncertain preferences provided by the DM.

$\begin{array}{ccccccc} & \mathrm{B} & \mathrm{C} & \mathrm{F} & \mathrm{R} & \mathrm{P} & \mathrm{V} \\ \mathrm{B} & 0 & 0 & 0 & 0 & 1 & 0 \\ \mathrm{C} & 1 & 0 & 0 & 1 & 1 & 0 \\ \mathrm{~F} & 1 & 1 & 0 & 1 & 1 & 0 \\ \mathrm{R} & 1 & 0 & 0 & 0 & 1 & 0 \\ \mathrm{P} & 0 & 0 & 0 & 0 & 0 & 0 \\ \mathrm{~V} & 1 & 1 & 1 & 1 & 1 & 0\end{array}$

2. $\succsim^{N} \subseteq \succsim_{L}^{N} \subseteq \succsim_{L}^{R} \subseteq \succsim_{L}^{P} \subseteq \succsim^{P}$

To illustrate this proposition, in Tables 6(a) and 6(b) we present the "classical" necessary and possible preference relations (i.e., for certain preference information only). Analyzing Tables

Table 6: Necessary and possible preference relations

\begin{tabular}{|c|c|c|c|c|c|c|c|c|c|c|c|c|c|}
\hline $\begin{array}{l}\text { (a) } \\
\text { tion }\end{array}$ & & & & & & rela- & $\begin{array}{l}\text { (b) } \\
\succsim^{P} .\end{array}$ & & & & & & atio \\
\hline & B & $\mathrm{C}$ & $\mathrm{F}$ & $\mathrm{R}$ & $\mathrm{P}$ & $\mathrm{V}$ & & B & $\mathrm{C}$ & $\mathrm{F}$ & $\mathrm{R}$ & $\mathrm{P}$ & $\mathrm{V}$ \\
\hline B & 1 & 0 & 0 & 0 & 0 & 0 & B & 1 & 1 & 1 & 1 & 1 & 1 \\
\hline $\mathrm{C}$ & 0 & 1 & 0 & 0 & 0 & 0 & $\mathrm{C}$ & 1 & 1 & 0 & 1 & 1 & 1 \\
\hline $\mathrm{F}$ & 0 & 1 & 1 & 0 & 0 & 0 & $\mathrm{~F}$ & 1 & 1 & 1 & 1 & 1 & 1 \\
\hline $\mathrm{R}$ & 0 & 0 & 0 & 1 & 0 & 0 & $\mathrm{R}$ & 1 & 1 & 1 & 1 & 1 & 1 \\
\hline $\mathrm{P}$ & 0 & 0 & 0 & 0 & 1 & 0 & $\mathrm{P}$ & 1 & 1 & 1 & 1 & 1 & 0 \\
\hline V & 0 & 0 & 0 & 0 & 1 & 1 & $\mathrm{~V}$ & 1 & 1 & 1 & 1 & 1 & 1 \\
\hline
\end{tabular}

4, 5 and 6, and considering, e.g., pair of managers $(F, C)$, it is easy to observe that all the inclusions from point 2 hold. Indeed, $F \succsim^{N} C, F \succsim_{L}^{N} C, F \succsim_{L}^{R} C, F \succsim_{L}^{P} C$ and $F \succsim^{P} C$. Of course, the opposite inclusions do not hold. For example, $C \succsim^{P} V$ but $\operatorname{not}\left(C \succsim_{L}^{P} V\right) ; B \succsim_{L}^{P} F$ but $\operatorname{not}\left(B \succsim_{L}^{R} F\right) ; C \succsim_{L}^{R} P$ but $\operatorname{not}\left(C \succsim_{L}^{N} P\right)$. Finally, $V \succsim_{L}^{N} C$ but $\operatorname{not}\left(V \succsim^{N} C\right)$.

3. For all $a, b \in A, a \succsim_{L}^{N} b$ or $b \succsim_{L}^{P} a$.

For example, given pair $(V, C), V \succsim_{L}^{N} C$ but $\operatorname{not}\left(C \succsim_{L}^{P} V\right)$, while for $(C, B)$ we have $C \succsim_{L}^{P} B$ 
but $\operatorname{not}\left(B \succsim_{L}^{N} C\right)$

4. For all $a, b, c \in A$, if $a \succsim^{N} b$ and $b \succsim_{L}^{N} c$, then $a \succsim_{L}^{N} c$.

For example, considering $F, C$ and $R$, we have that $F \succsim^{N} C, C \succsim_{L}^{N} R$ and, consequently, $F \succsim_{L}^{N} R$

5. For all $a, b, c \in A$, if $a \succsim_{L}^{N} b$ and $b \succsim^{N} c$, then $a \succsim_{L}^{N} c$.

For example, considering $V, F$ and $C$, we have that $V \succsim_{L}^{N} F, F \succsim^{N} C$ and, consequently, $V \succsim_{L}^{N} C$

6. For all $a, b, c \in A$, if $a \succsim^{N} b$ and $b \succsim_{L}^{P} c$, then $a \succsim_{L}^{P} c$.

For example, considering $V, P$ and $F$, we have that $V \succsim^{N} P, P \succsim_{L}^{P} F$ and, consequently, $V \succsim_{L}^{P} F$

7. For all $a, b, c \in A$, if $a \succsim_{L}^{P} b$ and $b \succsim^{N} c$, then $a \succsim_{L}^{P} c$.

For example, considering $B, F$ and $C$, we have that $B \succsim_{L}^{P} F, F \succsim^{N} C$ and, consequently, $B \succsim_{L}^{P} C$

\subsection{Discussion of computational details.}

To discuss computational details concerning calculation of necessary and possible preference relations, both in the case of only certain preference information, and in the case of certain and uncertain preference information, as well as $R A I$ and $P W I$ indices of the stochastic ordinal regression, we shall present our procedure using a small set of compatible value functions and a small set of compatible probability distributions on the sample of compatible value functions.

To check if there exists at least one value function compatible with the certain preference information provided by the DM, we have to solve the LP problem (1). Observe that all three criteria are of gain type (i.e., the greater the evaluation on such a criterion, the better) and, consequently, the corresponding marginal value functions are non-decreasing with respect to performances. The variables of this LP problem are the marginal values for all different performances of the 6 sales managers on the 3 considered criteria, as shown in Table 7.

Using the variables shown in Table 7 and considering the certain preference information provided 
Table 7: The variables of the LP problem (1), corresponding to marginal values for all different performances of considered alternatives on criteria $g_{1}, g_{2}, g_{3}$.

$\begin{array}{ccc}u_{1} & u_{2} & u_{3} \\ u_{1}(6) & u_{2}(15) & u_{3}(0) \\ u_{1}(9) & u_{2}(18) & u_{3}(25) \\ u_{1}(26) & u_{2}(40) & u_{3}(28) \\ u_{1}(28) & u_{2}(43) & u_{3}(44) \\ u_{1}(35) & u_{2}(62) & u_{3}(88) \\ u_{1}(62) & & u_{3}(100)\end{array}$

by the DM, the LP problem (1) can be formulated in the following way:

$$
\begin{aligned}
& \varepsilon_{\text {certain }}=\max \varepsilon, \text { subject to, } \\
& u_{1}(62)+u_{2}(43)+u_{3}(0) \geq u_{1}(6)+u_{2}(15)+u_{3}(100)+\varepsilon, \quad V \succ P, \\
& \left.\begin{array}{l}
u_{1}(62)+u_{2}(43)+u_{3}(0)-u_{1}(6)-u_{2}(15)-u_{3}(100) \geq \\
\geq u_{1}(35)+u_{2}(62)+u_{3}(25)-u_{1}(26)-u_{2}(40)-u_{3}(44)+\varepsilon, \\
u_{1}(35)+u_{2}(62)+u_{3}(25) \geq u_{1}(26)+u_{2}(40)+u_{3}(44)+\varepsilon,
\end{array}\right\}\left[(V, P) \succ^{*}(F, C)\right], \\
& u_{1}(62)+u_{2}(62)+u_{3}(100)=1, \quad u_{1}(6)=u_{2}(15)=u_{3}(0)=0 \text {, } \\
& u_{1}(6) \leq u_{1}(9) \leq u_{1}(26) \leq u_{1}(28) \leq u_{1}(35) \leq u_{1}(62), \\
& u_{2}(15) \leq u_{2}(18) \leq u_{2}(40) \leq u_{2}(43) \leq u_{2}(62), \\
& u_{3}(0) \leq u_{3}(25) \leq u_{3}(28) \leq u_{3}(44) \leq u_{3}(88) \leq u_{3}(100) .
\end{aligned}
$$

Since $\varepsilon_{\text {certain }}>0$, we conclude that the polyhedron of compatible value functions is not empty. Therefore, it is meaningful to compute necessary and possible preference relations $\succsim^{N}$ and $\succsim^{P}$. These relations were already calculated and shown in Table 6. To explain these calculations, let us consider the pair of sales managers Calvet and Petron $(C, P)$. To check if Calvet is possibly preferred to Petron, i.e., $C \succsim^{P} P$, we have to solve the LP problem obtained from LP (4) by adding the constraint $U(C) \geq U(P)$, i.e.:

$$
u_{1}(26)+u_{2}(40)+u_{3}(44) \geq u_{1}(6)+u_{2}(15)+u_{3}(100) .
$$


Since $\varepsilon^{P}=\max \varepsilon>0$, there exists a compatible value function $U$, such that $U(C) \geq U(P)$, which permits to conclude that $C \succsim^{P} P$. Then, to check if Calvet is necessarily preferred to Petron, i.e., $C \succsim^{N} P$, we have to solve the LP problem obtained from LP (4) by adding the constraint $U(P) \geq U(C)+\varepsilon$, i.e.:

$$
u_{1}(6)+u_{2}(15)+u_{3}(100) \geq u_{1}(26)+u_{2}(40)+u_{3}(44)+\varepsilon .
$$

Since $\varepsilon^{N}=\max \varepsilon>0$, there exists a compatible value function $U$, such that $U(P)>U(C)$, which implies that it is not true that $U(C) \geq U(P)$ for all compatible value functions, i.e., $\operatorname{not}\left(C \succsim^{N} P\right)$. The necessary and possible preference relations for other pairs of sales managers have been calculated analogously.

Table 8: Marginal values of $U_{1}, U_{2}, U_{3}, U_{4}$ for all different performances of alternatives on criteria $g_{1}, g_{2}, g_{3}$.

\begin{tabular}{|c|c|c|c|c|c|c|c|c|c|c|c|c|c|c|}
\hline & $U_{1}$ & $U_{2}$ & $U_{3}$ & $U_{4}$ & & $U_{1}$ & $U_{2}$ & $U_{3}$ & $U_{4}$ & & $U_{1}$ & $U_{2}$ & $U_{3}$ & $U_{4}$ \\
\hline${ }_{1}(6)$ & 0 & 0 & 0 & 0 & $u_{, 2}(15)$ & 0 & 0 & 0 & 0 & $u_{\cdot, 3}(0)$ & 0 & 0 & 0 & 0 \\
\hline , $1(9)$ & 1348 & 0885 & 1119 & 0015 & $u_{\cdot, 2}(18)$ & 0103 & 0193 & 1074 & 0664 & $u_{, 3,3}(25)$ & 1157 & 0.0192 & 0.0024 & 0214 \\
\hline$u_{\cdot, 1}(26)$ & 0.2259 & 0.1281 & 085 & 0.1697 & $u_{\cdot, 2}(40)$ & 0.0725 & 0.3192 & 0.2299 & 0.2554 & $u_{,, 3}(28)$ & 0.2206 & 0.0623 & 0.0126 & 0.0412 \\
\hline , $1(28)$ & 0.2951 & 0.1322 & 0.3052 & 0.2330 & $u_{\cdot, 2}(43)$ & 0.1160 & 0.4371 & 0.2303 & 0.3702 & $u_{,, 3}(44)$ & 0.2412 & 0.1806 & 0.0662 & 0.1877 \\
\hline ;,1(35) & 0.3613 & 0.1699 & 0.4036 & 0.2522 & $u_{\cdot, 2}(62)$ & 0.1515 & 0.4400 & 0.2649 & 0.3877 & $u_{,, 3}(88)$ & 0.2736 & 0.2122 & 0.1058 & 0.2202 \\
\hline ,1(62) & 0.5529 & 0.2257 & 0.4322 & 0.3422 & & & & & & $u_{,, 3}(100)$ & 0.2956 & 0.3343 & 0.3029 & 0.2701 \\
\hline
\end{tabular}

Now, we should define a probability distribution on $\mathcal{U}$ compatible with the uncertain preference information provided by the DM. Remember that $\mathcal{U}$ is the set of value functions $U$ compatible with the certain preference information provided by the DM. Unless $\mathcal{U}$ is empty, it is an infinite set, in general. Thus, we approximate the probability distribution on $\mathcal{U}$ by considering instead of $\mathcal{U}$ a well-distributed sample of value functions in $\mathcal{U}$, denoted by $\widehat{\mathcal{U}}$. To present this procedure in simple terms, we are considering $\widehat{\mathcal{U}}$ composed of only four value functions $U_{1}, U_{2}, U_{3}, U_{4}$ computed using the HAR method. They are shown in Table 8. Using the marginal values from Table 8, one can compute the comprehensive values of $U_{1}, U_{2}, U_{3}, U_{4}$ for each considered sales manager. These values are presented in Table 9. Analyzing the pairs of alternatives involved in the pieces of uncertain preference information (i.e., $C \succ_{L} R$ and $(V, F) \succ_{L}^{*}(C, R)$ ), one can observe that:

- $C$ is preferred to $R$ for $U_{3}$ and $U_{4}$, while $R$ is preferred to $C$ for $U_{1}$ and $U_{2}$; 
- $V$ is preferred to $F$ for $U_{1}, U_{2}$ and $U_{4}$, but not for $U_{3}$.

Table 9: Comprehensive values assigned to the six sales managers and respective rankings obtained with $U_{1}, U_{2}, U_{3}, U_{4}$.

\begin{tabular}{ccccccccc}
\multicolumn{3}{c}{ (a) Comprehensive values } & \multicolumn{5}{c}{ (b) Complete rankings } \\
& $U_{1}$ & $U_{2}$ & $U_{3}$ & $U_{4}$ & $U_{1}$ & $U_{2}$ & $U_{3}$ & $U_{4}$ \\
$\mathrm{~B}$ & 0.5259 & 0.2137 & 0.4252 & 0.3405 & $\mathrm{~V}(0.6689)$ & $\mathrm{R}(0.7405)$ & $\mathrm{F}(0.6708)$ & $\mathrm{V}(0.7123)$ \\
$\mathrm{C}$ & 0.5395 & 0.6279 & 0.5045 & 0.6128 & $\mathrm{~F}(0.6285)$ & $\mathrm{V}(0.6628)$ & $\mathrm{V}(0.6624)$ & $\mathrm{F}(0.6613)$ \\
$\mathrm{F}$ & 0.6285 & 0.6290 & 0.6708 & 0.6613 & $\mathrm{R}(0.5599)$ & $\mathrm{F}(0.6290)$ & $\mathrm{C}(0.5045)$ & $\mathrm{C}(0.6128)$ \\
$\mathrm{R}$ & 0.5599 & 0.7405 & 0.3825 & 0.6094 & $\mathrm{C}(0.5395)$ & $\mathrm{C}(0.6279)$ & $\mathrm{B}(0.4252)$ & $\mathrm{R}(0.6094)$ \\
$\mathrm{P}$ & 0.2955 & 0.3343 & 0.3029 & 0.2701 & $\mathrm{~B}(0.5259)$ & $\mathrm{P}(0.3343)$ & $\mathrm{R}(0.3825)$ & $\mathrm{B}(0.3405)$ \\
$\mathrm{V}$ & 0.6689 & 0.6628 & 0.6624 & 0.7123 & $\mathrm{P}(0.2955)$ & $\mathrm{B}(0.2137)$ & $\mathrm{P}(0.3029)$ & $\mathrm{P}(0.2701)$
\end{tabular}

To check if there exists a probability distribution on the set of value functions $U_{1}, U_{2}, U_{3}, U_{4}$, we have to solve the LP problem (2). A probability distribution on the set of sampled value functions is, in this case, a vector of four non-negative probabilities $\left(w\left(U_{1}\right), w\left(U_{2}\right), w\left(U_{3}\right), w\left(U_{4}\right)\right)$, such that $w\left(U_{1}\right)+w\left(U_{2}\right)+w\left(U_{3}\right)+w\left(U_{4}\right)=1$. The LP problem (2) that should be solved to verify the existence of a probability distribution compatible with the uncertain preference information provided by the $\mathrm{DM}$ is the following:

$$
\left.\begin{array}{ccc}
\varepsilon_{L}^{*}=\max \varepsilon, & \text { subject to } \\
w\left(U_{3}\right)+w\left(U_{4}\right) \geq w\left(U_{1}\right)+w\left(U_{2}\right)+\varepsilon, \quad C \succ_{L} R, & \\
w\left(U_{1}\right)+w\left(U_{2}\right)+w\left(U_{4}\right) \geq w\left(U_{3}\right)+\varepsilon, \quad(V, F) \succ_{L}^{*}(C, R), & \\
w\left(U_{1}\right)+w\left(U_{2}\right)+w\left(U_{3}\right)+w\left(U_{4}\right)=1, & {[U N C]} \\
w\left(U_{1}\right) \geq 0, w\left(U_{2}\right) \geq 0, w\left(U_{3}\right) \geq 0, w\left(U_{4}\right) \geq 0 . & {[U N N C]}
\end{array}\right\} E_{L}
$$

Since for the optimal solution of problem $(7) \varepsilon_{L}^{*}>0$, then the polyhedron of the probability distributions on the set of value functions $U_{1}, U_{2}, U_{3}, U_{4}$ compatible with the certain preference of the DM is not empty. Thus, one can sample a set of probability distributions compatible with the uncertain preferences of the DM. Let the sample be composed of five probability distributions $\mathbf{w}_{1}, \ldots, \mathbf{w}_{5}$ shown in Table 10. 
Table 10: Five sampled probability distributions $\mathbf{w}_{1}, \ldots, \mathbf{w}_{5}$, compatible with the uncertain preference information provided by the DM, and the representative probability distribution $\mathbf{w}^{*}$.

\begin{tabular}{ccccc} 
& $w\left(U_{1}\right)$ & $w\left(U_{2}\right)$ & $w\left(U_{3}\right)$ & $w\left(U_{4}\right)$ \\
$\mathbf{w}_{1}$ & 0.1794 & 0.2233 & 0.0135 & 0.5836 \\
$\mathbf{w}_{2}$ & 0.2094 & 0.1944 & 0.2121 & 0.3840 \\
$\mathbf{w}_{3}$ & 0.3761 & 0.0489 & 0.1045 & 0.4702 \\
$\mathbf{w}_{4}$ & 0.2023 & 0.0011 & 0.1814 & 0.6151 \\
$\mathbf{w}_{5}$ & 0.3390 & 0.0728 & 0.1723 & 0.4157 \\
\hline $\mathbf{w}^{*}$ & 0.2612 & 0.1081 & 0.1368 & 0.4937
\end{tabular}

Table 11: Rank acceptability indices of the six sales managers in the considered example.

$\begin{array}{ccccccc}a & R A I_{\mathbf{w}^{*}}(a, 1) & R A I_{\mathbf{w}^{*}}(a, 2) & R A I_{\mathbf{w}^{*}}(a, 3) & R A I_{\mathbf{w}^{*}}(a, 4) & R A I_{\mathbf{w}^{*}}(a, 5) & R A I_{\mathbf{w}^{*}}(a, 6) \\ \mathrm{B} & 0 & 0 & 0 & 0.1368 & 0.7550 & 0.1081 \\ \mathrm{C} & 0 & 0 & 0.6305 & 0.3694 & 0 & 0 \\ \mathrm{~F} & 0.1368 & 0.7550 & 0.1081 & 0 & 0 & 0 \\ \mathrm{R} & 0.1081 & 0 & 0.2612 & 0.4937 & 0.1368 & 0 \\ \mathrm{P} & 0 & 0 & 0 & 0 & 0.1081 & 0.8918 \\ \mathrm{~V} & 0.7550 & 0.2449 & 0 & 0 & 0 & 0\end{array}$

Table 12: Pairwise winning indices $P W I_{\mathbf{w}^{*}}$ for the sales managers in the considered example.

$\begin{array}{ccccccc} & \mathrm{B} & \mathrm{C} & \mathrm{F} & \mathrm{R} & \mathrm{P} & \mathrm{V} \\ \mathrm{B} & 0 & 0 & 0 & 0.1368 & 0.8918 & 0 \\ \mathrm{C} & 1.00 & 0 & 0 & 0.6305 & 1.00 & 0 \\ \mathrm{~F} & 1.00 & 1.00 & 0 & 0.8918 & 1.00 & 0.1368 \\ \mathrm{R} & 0.8631 & 0.3694 & 0.1081 & 0 & 1.00 & 0.1081 \\ \mathrm{P} & 0.1081 & 0 & 0 & 0 & 0 & 0 \\ \mathrm{~V} & 1.00 & 1.00 & 0.8631 & 0.8918 & 1.00 & 0\end{array}$

Now, let us explain how $R A I \mathrm{~s}$ and $P W I$ s presented in Tables 11 and 12, respectively, are computed. Let us consider the rank acceptability index of Varlot for the first position. In Table 11 , we note that $R A I_{\mathbf{w}^{*}}(V, 1)=0.755$. This means that the sum of probabilities assigned 
to the sampled value functions for which Varlot attains the first rank is equal to $0.755 \%$. Indeed, looking at Table 9(b), one can observe that Varlot attains the first rank for value functions $U_{1}$ and $U_{4}$. Consequently, $R A I_{\mathbf{w}^{*}}(V, 1)=\mathbf{w}^{*}\left(U_{1}\right)+\mathbf{w}^{*}\left(U_{4}\right)=0.2612+0.4937=0.755$. The same reasoning can be applied when computing the pairwise winning index for Calvet and Frechet. In Table 12, we note that $P W I_{\mathbf{w}^{*}}(C, R)=0.6305$ and $P W I_{\mathbf{w}^{*}}(R, C)=0.3694$. Indeed, remembering that $P W I_{\mathbf{w}^{*}}(a, b)$ is the probability with which $a$ is preferred to $b$, based on Table $9(\mathrm{~b})$, one can check that $C$ is preferred to $R$ for $U_{3}$ and $U_{4}$, while $R$ is preferred to $C$ for $U_{1}$ and $U_{2}$. Consequently, $P W I_{\mathbf{w}^{*}}(C, R)=\mathbf{w}^{*}\left(U_{3}\right)+\mathbf{w}^{*}\left(U_{4}\right)=0.1368+0.4937=0.6305$, while $P W I_{\mathbf{w}^{*}}(R, C)=\mathbf{w}^{*}\left(U_{1}\right)+\mathbf{w}^{*}\left(U_{2}\right)=0.2612+0.1081=0.3694$.

To end this example, where only four value functions $U_{1}, \ldots, U_{4}$ were sampled in the space of all value functions compatible with the certain preference information, let us compute the probabilistic necessary and possible preference relations $\succsim_{L}^{N}$ and $\succsim_{L}^{P}$.

In order to check if, for example, $V$ is probabilistically possibly preferred to $R$, which is denoted by $V \succsim_{L}^{P} R$, we have to verify if there exists at least one probability distribution over value functions $U_{1}, \ldots, U_{4}$, compatible with the uncertain preferences of the DM, such that the probability of the preference of $V$ over $R$ is no lower than the probability of the preference of $R$ over $V$. For this, we have to solve the LP problem (7) with an additional constraint expressing the requirement that the probability of the value functions for which $V$ is preferred to $R$, i.e., $U_{1}, U_{3}, U_{4}$, is not smaller than the probability of the value function for which $R$ is preferred to $V$, i.e., $U_{2}$ :

$$
w\left(U_{1}\right)+w\left(U_{3}\right)+w\left(U_{4}\right) \geq w\left(U_{2}\right)
$$

We conclude that $V \succsim_{L}^{P} R$ if the set of constraints is feasible and $\varepsilon_{L}^{P}>0$, where $\varepsilon_{L}^{P}=\max \varepsilon$, subject to the constraints $E_{L}$ and (8). Since $\varepsilon_{L}^{P}=1$ (for $w_{1}=w_{2}=w_{3}=0, w_{4}=1$ ), we have $V \succsim_{L}^{P} R$.

To check if $V$ is probabilistically necessarily preferred to $R$, which is denoted by $V \succsim_{L}^{N} R$, we have to verify in turn if the probability of the preference of $V$ over $R$ is no lower than the probability of the preference of $R$ over $V$. This is equivalent to say that there is no probability distribution for which the probability assigned to the value function preferring $R$ over $V$ (i.e., $U_{2}$ ) is greater than the probability assigned to the value functions preferring $V$ over $R$ (i.e., $U_{1}, U_{3}, U_{4}$ ). For this, we have 
to solve the LP problem (7) with an additional constraint expressing the above requirement:

$$
w\left(U_{2}\right) \geq w\left(U_{1}\right)+w\left(U_{3}\right)+w\left(U_{4}\right)+\varepsilon .
$$

We conclude that $V \succsim_{L}^{N} R$ if the set of constraints is not feasible or $\varepsilon_{L}^{N} \leq 0$, where $\varepsilon_{L}^{N}=\max \varepsilon$, subject to the constraints $E_{L}$ and (9). Since $\varepsilon_{L}^{N}=0$ (for $w_{1}=w_{4}=0, w_{2}=w_{4}=0.5$ ), we have $V \succsim_{L}^{N} R$

Analogously, one can compute the probabilistic necessary and possible preference relations $\succsim_{L}^{N}$ and $\succsim_{L}^{P}$ for all the pairs of sales managers. The results are shown in Table 13 .

To conclude the example, let us remind that computation of probability distributions $\mathbf{w}$, their barycenter $\mathbf{w}^{*}, R A I_{\mathbf{w}^{*}}, P W I_{\mathbf{w}^{*}}$, as well as $\succsim_{L}^{P}$ and $\succsim_{L}^{N}$, have been performed taking into account a small sample of compatible value functions and a small number of probability distributions on this sample. This was done for the sake of a didactic presentation, however, in order to get a reasonable approximation of the analogous results on the whole set of compatible value functions $\mathcal{U}$ and probability distribution on $\mathcal{U}$, it is necessary to consider much larger samples of compatible value functions and probability distributions, for example, 10,000 for both of them, as in Subsection 3.1.

Table 13: Probabilistic preference relations in the space of probability distributions for $U_{1}, \ldots, U_{4}$.

(a) Probabilistic necessary preference relation $\succsim_{L}^{N}$.

$\begin{array}{ccccccc} & \mathrm{B} & \mathrm{C} & \mathrm{F} & \mathrm{R} & \mathrm{P} & \mathrm{V} \\ \mathrm{B} & 1 & 0 & 0 & 0 & 1 & 0 \\ \mathrm{C} & 1 & 1 & 0 & 1 & 1 & 0 \\ \mathrm{~F} & 1 & 1 & 1 & 1 & 1 & 0 \\ \mathrm{R} & 1 & 0 & 0 & 1 & 1 & 0 \\ \mathrm{P} & 0 & 0 & 0 & 0 & 1 & 0 \\ \mathrm{~V} & 1 & 1 & 1 & 1 & 1 & 1\end{array}$

(b) Probabilistic possible

preference relation $\succsim_{L}^{P}$.

$\begin{array}{ccccccc} & \mathrm{B} & \mathrm{C} & \mathrm{F} & \mathrm{R} & \mathrm{P} & \mathrm{V} \\ \mathrm{B} & 1 & 0 & 0 & 1 & 1 & 0 \\ \mathrm{C} & 1 & 1 & 0 & 1 & 1 & 0 \\ \mathrm{~F} & 1 & 1 & 1 & 1 & 1 & 1 \\ \mathrm{R} & 1 & 0 & 0 & 1 & 1 & 0 \\ \mathrm{P} & 0 & 0 & 0 & 0 & 1 & 0 \\ \mathrm{~V} & 1 & 1 & 1 & 1 & 1 & 1\end{array}$




\section{Conclusions}

In this paper, we introduced Subjective Stochastic Ordinal Regression (SSOR). SSOR is a generalization of the existing ordinal regression approaches, which are:

- Ordinal Regression, representing the preference information of the DM by means of a single compatible additive value function;

- Robust Ordinal Regression, representing the preference information of the DM by means of the whole set of compatible additive value functions;

- Stochastic Ordinal Regression, considering a probability distribution exogenously given (very often a uniform distribution) on the space of compatible additive value functions.

Differently from all these methods taking into account only certain preference information, in SSOR uncertain preference information supplied by the DM is also taken into account. Indeed, in real world applications, the DM could wish to provide not only the certain preferences, but also some uncertain ones, stating, e.g., that the preference of alternative $a$ over alternative $b$ is more credible than the preference of $b$ over $a$. The uncertain preference information permits to build a probability distribution over the set of preference models compatible with the certain preference information provided by the DM.

Although we implemented SSOR for the case of preference modeling by an additive value functions, this approach can be applied to other preference models already considered within OR and ROR, such as outranking relation used in ELECTRE or PROMETHEE methods [6, 9, 17, 29, 40], or models incorporating interactions between criteria $[1,3,7,20,24]$. Moreover, SSOR is also applicable to multiple criteria sorting methods where alternatives have to be assigned to pre-defined and preferentially ordered classes $[8,23,27,36]$ as well as to group decision problems. Finally, the concept of uncertain preference information can be used to enrich the explanations of the obtained recommendation [28]. 


\section{Acknowledgments}

The first two authors wish to acknowledge funding by the "FIR of the University of Catania BCAEA3 New developments in Multiple Criteria Decision Aiding (MCDA) and their application to territorial competitiveness". The third author acknowledges financial support from the Polish Ministry of Science and Higher Education under the Iuventus Plus program in grant number IP2015 029674 0296/IP5/2016/74.

[1] S. Angilella, S. Corrente, S. Greco, and R. Słowiński. MUSA-INT: Multicriteria customer satisfaction analysis with interacting criteria. Omega, 42(1):189 - 200, 2014.

[2] S. Angilella, S. Corrente, S. Greco, and R. Słowiński. Robust Ordinal Regression and Stochastic Multiobjective Acceptability Analysis in multiple criteria hierarchy process for the Choquet integral preference model. Omega, (63):154-169, 2016.

[3] S. Angilella, S. Greco, and B. Matarazzo. Non-additive robust ordinal regression: A multiple criteria decision model based on the choquet integral. European Journal of Operational Research, 201(1):277 - 288, 2010.

[4] S. Aydin, C. Kahraman, and İ. Kaya. A new fuzzy multicriteria decision making approach: An application for european quality award assessment. Knowledge-Based Systems, 32:37-46, 2012.

[5] C. Bana e Costa and J.-C. Vansnick. MACBETH-An interactive path towards the construction of cardinal value functions. International Transactions in Operational Research, 1(4):489-500, 1994.

[6] J.P. Brans and Ph. Vincke. A preference ranking organisation method: The PROMETHEE method for MCDM. Management Science, 31(6):647-656, 1985.

[7] G. Choquet. Theory of capacities. Annales de l'institut Fourier, 5:131-295, 1954.

[8] S. Corrente, M. Doumpos, S. Greco, R. Słowiński, and C. Zopounidis. Multiple criteria hierarchy process for sorting problems based on ordinal regression with additive value functions. Annals of Operations Research, (in press), 2015. 
[9] S. Corrente, J.R. Figueira, and S. Greco. Dealing with interaction between bipolar multiple criteria preferences in PROMETHEE methods. Annals of Operations Research, 217(1):137-164, 2014 .

[10] S. Corrente, S. Greco, M. Kadziński, and R. Słowiński. Robust ordinal regression in preference learning and ranking. Machine Learning, 93:381-422, 2013.

[11] S. Corrente, S. Greco, M. Kadziński, and R. Słowiński. Robust Ordinal Regression. Wiley Encyclopedia of Operations Research and Management Science, pages 1-10, 2014.

[12] B. De Baets and J. Fodor. Preference modelling, a matter of degree. In M. Ehrgott, J. Figueira, and S. Greco, editors, Trends in multiple criteria decision analysis, pages 141-176. Springer US, 2010.

[13] M. Doumpos, C. Zopounidis, and E. Galariotis. Inferring robust decision models in multicriteria classification problems: An experimental analysis. European Journal of Operational Research, 236(2):601-611, 2014.

[14] K. Eliaz and E.A. Ok. Indifference or indecisiveness? Choice-theoretic foundations of incomplete preferences. Games and Economic Behavior, 56:61-86, 2006.

[15] N. Fenton and W. Wang. Risk and confidence analysis for fuzzy multicriteria decision making. Knowledge-Based Systems, 19(6):430-437, 2006.

[16] J.R. Figueira, S. Greco, and M. Ehrgott. Multiple Criteria Decision Analysis: State of the Art Surveys. Springer, Berlin, 2005.

[17] J.R. Figueira, S. Greco, R. Słowiński, and B. Roy. An overview of ELECTRE methods and their recent extensions. Journal of Multi-Criteria Decision Analysis, 20:61-85, 2013.

[18] T. Galaabaatar and E. Karni. Subjective expected utility with incomplete preferences. Econometrica, 81:255-284, 2013.

[19] A. Giarlotta and S. Greco. Necessary and possible preference structures. Journal of Mathematical Economics, 49(2):163-172, 2013. 
[20] M. Grabisch. The application of fuzzy integrals in multicriteria decision making. European Journal of Operational Research, 89(3):445-456, 1996.

[21] S. Greco, B. Matarazzo, and R. Słowiński. Rough sets theory for multicriteria decision analysis. European Journal of Operational Research, 129(1):1-47, 2001.

[22] S. Greco, V. Mousseau, and R. Słowiński. Ordinal regression revisited: multiple criteria ranking using a set of additive value functions. European Journal of Operational Research, 191(2):416436, 2008.

[23] S. Greco, V. Mousseau, and R. Słowiński. Multiple criteria sorting with a set of additive value functions. European Journal of Operational Research, 207(3):1455-1470, 2010.

[24] S. Greco, V. Mousseau, and R. Słowiński. Robust ordinal regression for value functions handling interacting criteria. European Journal of Operational Research, 239(3):711-730, 2014.

[25] E. Jacquet-Lagrèze and Y. Siskos. Assessing a set of additive utility functions for multicriteria decision-making, the UTA method. European Journal of Operational Research, 10(2):151-164, 1982.

[26] E. Jacquet-Lagrèze and Y. Siskos. Preference disaggregation: 20 years of MCDA experience. European Journal of Operational Research, 130(2):233-245, 2001.

[27] M. Kadziński, K. Ciomek, and R. Słowiński. Modeling assignment-based pairwise comparisons within integrated framework for value-driven multiple criteria sorting. European Journal of Operational Research, 241(3):830 - 841, 2015.

[28] M. Kadziński, S. Corrente, S. Greco, and R. Słowiński. Preferential reducts and constructs in robust multiple criteria ranking and sorting. OR Spectrum, 36(4):1021-1053, 2014.

[29] M. Kadziński, S. Greco, and R. Słowiński. Extreme ranking analysis in robust ordinal regression. Omega, 40(4):488-501, 2012.

[30] M. Kadziński, R. Słowiński, and S. Greco. Multiple criteria ranking and choice with all compatible minimal cover sets of decision rules. Knowledge-Based Systems, 89:569-583, 2015. 
[31] M. Kadziński and T. Tervonen. Robust multi-criteria ranking with additive value models and holistic pair-wise preference statements. European Journal of Operational Research, 228(1):169180, 2013.

[32] M. Kadziński and T. Tervonen. Stochastic ordinal regression for multiple criteria sorting problems. Decision Support Systems, 55(11):55-66, 2013.

[33] C. Kahraman, B. Öztayşi, İ. Sarı, and E. Turanoğlu. Fuzzy analytic hierarchy process with interval type-2 fuzzy sets. Knowledge-Based Systems, 59:48-57, 2014.

[34] R.L. Keeney and H. Raiffa. Decisions with multiple objectives: Preferences and value tradeoffs. J. Wiley, New York, 1993.

[35] R. Lahdelma, J. Hokkanen, and P. Salminen. SMAA - stochastic multiobjective acceptability analysis. European Journal of Operational Research, 106(1):137-143, 1998.

[36] J. Liu, X. Liao, and J. Yang. A group decision-making approach based on evidential reasoning for multiple criteria sorting problem with uncertainty. European Journal of Operational Research, $246(3): 858-873,2015$.

[37] S. Minardi and A. Savochkin. Preference with grades of indecisiveness. Journal of Economic Theory, 155:300-331, 2015.

[38] V. Mousseau, L.C. Dias, and J.R. Figueira. Dealing with inconsistent judgments in multiple criteria sorting models. 4OR, 4(2):145-158, 2006.

[39] V. Mousseau, J. Figueira, L. Dias, C. Gomes da Silva, and J. Climaco. Resolving inconsistencies among constraints on the parameters of an MCDA model. European Journal of Operational Research, 147(1):72-93, 2003.

[40] B. Roy. The outranking approach and the foundations of ELECTRE methods. Theory and Decision, 31:49-73, 1991.

[41] T.L Saaty. How to make a decision: the Analytic Hierarchy Process. European Journal of Operational Research, 48(1):9-26, 1990. 
[42] Y. Siskos and E. Grigoroudis. New Trends in Aggregation-Disaggregation Approaches. In C. Zopounidis and P. Pardalos, editors, Handbook of Multicriteria Analysis, pages 189-214, 2010.

[43] R.L. Smith. Efficient Monte Carlo procedures for generating points uniformly distributed over bounded regions. Operations Research, 32:1296-1308, 1984.

[44] T. Tervonen, G. Van Valkenhoef, N. Bastürk, and D. Postmus. Hit-And-Run enables efficient weight generation for simulation-based multiple criteria decision analysis. European Journal of Operational Research, 224:552-559, 2013.

[45] G. Van Valkenhoef, T. Tervonen, and D. Postmus. Notes on "Hit-And-Run enables efficient weight generation for simulation-based multiple criteria decision analysis". European Journal of Operational Research, 239:865-867, 2014.

\section{Appendix}

\section{Proof of Proposition 2.1}

1. Let $a, b \in A$ such that $\operatorname{not}\left(a \succsim_{L}^{\mathbf{w}} b\right)$. This means that for the considered compatible probability distribution w, $\sum_{t: U_{t}(a)>U_{t}(b)} w\left(U_{t}\right)<\sum_{t: U_{t}(b)>U_{t}(a)} w\left(U_{t}\right)$; therefore $b \succsim_{L}^{\mathbf{w}} a$.

2.1 Let $a, b \in A$ such that $a \succsim^{N} b$. Then, for all $U_{t}, t=1, \ldots, s_{v}, U_{t}(a) \geq U_{t}(b)$. Consequently, for all compatible probability distributions, $\sum_{t: U_{t}(b)>U_{t}(a)} w\left(U_{t}\right)=0$ because $\left\{t: U_{t}(b)>U_{t}(a)\right\}=\emptyset$.

Therefore, being $w(\cdot) \geq 0$, we have the thesis $\sum_{t: U_{t}(a)>U_{t}(b)} w\left(U_{t}\right) \geq \sum_{t: U_{t}(b)>U_{t}(a)} w\left(U_{t}\right)=0$.

2.2 It is obvious that $a \succsim_{L}^{N} b$ implies $a \succsim_{L}^{R} b$. In fact, if the preference of $a$ over $b$ is more credible than the preference of $b$ over $a$ for all compatible probability distributions, then it is true for whichever of them.

2.3 Analogously, it is obvious that $a \succsim_{L}^{R} b$ implies $a \succsim_{L}^{P} b$ for the definition of the binary relation $\succsim_{L}^{P}$ 
2.4 Let $a, b \in A$ such that $a \succsim_{L}^{P} b$ and let us suppose, for contradiction, that $\operatorname{not}\left(a \succsim^{P} b\right)$. This means that for all $U_{t}, t=1, \ldots, s_{v}, U_{t}(b)>U_{t}(a)$. Consequently, for all compatible probability distributions, we would have $\sum_{t: U_{t}(a)>U_{t}(b)} w\left(U_{t}\right)=0$ and $\sum_{t: U_{t}(b)>U_{t}(a)} w\left(U_{t}\right)=1$ being in contradiction with the hypothesis that $a \succsim_{L}^{P} b$, that is $\sum_{t: U_{t}(a)>U_{t}(b)} w\left(U_{t}\right) \geq \sum_{t: U_{t}(b)>U_{t}(a)} w\left(U_{t}\right)$. 3 Let $a, b \in A$ such that $\operatorname{not}\left(a \succsim_{L}^{N} b\right)$. This means that there exists at least one compatible probability distribution for which $\sum_{t: U_{t}(a)>U_{t}(b)} w\left(U_{t}\right)<\sum_{t: U_{t}(b)>U_{t}(a)} w\left(U_{t}\right)$. As a consequence, $b \succsim_{L}^{P} a$.

4 Let $a, b, c \in A$ such that $a \succsim^{N} b$ and $b \succsim_{L}^{N} c$. Since $a \succsim^{N} b$, we have $U_{t}(a) \geq U_{t}(b)$ for all $U_{t}, t=1, \ldots, s_{v}$, so that $\left\{t: U_{t}(b)>U_{t}(c)\right\} \subseteq\left\{t: U_{t}(a)>U_{t}(c)\right\}$ and, therefore, for all compatible probability distributions $\sum_{t: U_{t}(a)>U_{t}(c)} w\left(U_{t}\right) \geq \sum_{t: U_{t}(b)>U_{t}(c)} w\left(U_{t}\right)$, all $w(\cdot)$ being nonnegative. For the same reason, $\left\{t: U_{t}(c)>U_{t}(a)\right\} \subseteq\left\{t: U_{t}(c)>U_{t}(b)\right\}$ and, consequently, for all compatible probability distributions $\mathbf{w}, \sum_{t: U_{t}(c)>U_{t}(b)} w\left(U_{t}\right) \geq \sum_{t: U_{t}(c)>U_{t}(a)} w\left(U_{t}\right)$. Therefore, for all compatible probability distributions,

$$
\sum_{t: U_{t}(a)>U_{t}(c)} w\left(U_{t}\right) \geq \sum_{t: U_{t}(b)>U_{t}(c)} w\left(U_{t}\right) \geq \sum_{t: U_{t}(c)>U_{t}(b)} w\left(U_{t}\right) \geq \sum_{t: U_{t}(c)>U_{t}(a)} w\left(U_{t}\right)
$$

and, consequently, $\sum_{t: U_{t}(a)>U_{t}(c)} w\left(U_{t}\right) \geq \sum_{t: U_{t}(c)>U_{t}(a)} w\left(U_{t}\right)$ being the thesis.

5 Let $a, b \in A$ such that $a \succsim_{L}^{N} b$ and $b \succsim^{N} c$. Since $b \succsim^{N} c$, we have $U_{t}(b) \geq U_{t}(c)$ for all $U_{t}, t=$ $1, \ldots, s_{v}$, so that $\left\{t: U_{t}(a)>U_{t}(b)\right\} \subseteq\left\{t: U_{t}(a)>U_{t}(c)\right\}$ and, consequently, for all compatible probability distributions $\mathbf{w}, \sum_{t: U_{t}(a)>U_{t}(c)} w\left(U_{t}\right) \geq \sum_{t: U_{t}(a)>U_{t}(b)} w\left(U_{t}\right)$ because all $w(\cdot)$ are nonnegative. For the same reason, $\left\{t: U_{t}(c)>U_{t}(a)\right\} \subseteq\left\{t: U_{t}(b)>U_{t}(a)\right\}$ and, consequently, for all compatible probability distributions $\mathbf{w}, \sum_{t: U_{t}(b)>U_{t}(a)} w\left(U_{t}\right) \geq \sum_{t: U_{t}(c)>U_{t}(a)} w\left(U_{t}\right)$. Therefore, for all compatible probability distributions,

$$
\sum_{t: U_{t}(a)>U_{t}(c)} w\left(U_{t}\right) \geq \sum_{t: U_{t}(a)>U_{t}(b)} w\left(U_{t}\right) \geq \sum_{t: U_{t}(b)>U_{t}(a)} w\left(U_{t}\right) \geq \sum_{t: U_{t}(c)>U_{t}(a)} w\left(U_{t}\right)
$$

and, consequently, $\sum_{t: U_{t}(a)>U_{t}(c)} w\left(U_{t}\right) \geq \sum_{t: U_{t}(c)>U_{t}(a)} w\left(U_{t}\right)$ being the thesis. 
6 Let $a, b, c \in A$ such that $a \succsim^{N} b$ and $b \succsim_{L}^{P} c$. Since $a \succsim^{N} b$ we have $U_{t}(a) \geq U_{t}(b)$ for all $U_{t}, t=$ $1, \ldots, s_{v}$, so that $\left\{t: U_{t}(b)>U_{t}(c)\right\} \subseteq\left\{t: U_{t}(a)>U_{t}(c)\right\}$ and, therefore, for all compatible probability distributions $\mathbf{w}, \sum_{t: U_{t}(a)>U_{t}(c)} w\left(U_{t}\right) \geq \sum_{t: U_{t}(b)>U_{t}(c)} w\left(U_{t}\right)$ because all $w(\cdot)$ are nonnegative. For the same reason, $\left\{t: U_{t}(c)>U_{t}(a)\right\} \subseteq\left\{t: U_{t}(c)>U_{t}(b)\right\}$ and, consequently, for all compatible probability distributions $\sum_{t: U_{t}(c)>U_{t}(b)} w\left(U_{t}\right) \geq \sum_{t: U_{t}(c)>U_{t}(a)} w\left(U_{t}\right)$. Therefore, for the compatible probability distribution for which $b \succsim_{L}^{P} c$ we have,

$$
\sum_{t: U_{t}(a)>U_{t}(c)} w\left(U_{t}\right) \geq \sum_{t: U_{t}(b)>U_{t}(c)} w\left(U_{t}\right) \geq \sum_{t: U_{t}(c)>U_{t}(b)} w\left(U_{t}\right) \geq \sum_{t: U_{t}(c)>U_{t}(a)} w\left(U_{t}\right)
$$

and, consequently, for this compatible probability distribution $\sum_{t: U_{t}(a)>U_{t}(c)} w\left(U_{t}\right) \geq \sum_{t: U_{t}(c)>U_{t}(a)} w\left(U_{t}\right)$ being the thesis.

7 Let $a, b \in A$ such that $a \succsim_{L}^{P} b$ and $b \succsim^{N} c$. Since $b \succsim^{N} c$, we have $U_{t}(b) \geq U_{t}(c)$ for all $U_{t}, t=$ $1, \ldots, s_{v}$, so that $\left\{t: U_{t}(a)>U_{t}(b)\right\} \subseteq\left\{t: U_{t}(a)>U_{t}(c)\right\}$ and, consequently, $\sum_{t: U_{t}(a)>U_{t}(c)} w\left(U_{t}\right) \geq$ $\sum_{t: U_{t}(a)>U_{t}(b)} w\left(U_{t}\right)$ because all $w(\cdot)$ are non-negative. For the same reason, $\left\{t: U_{t}(c)>U_{t}(a)\right\} \subseteq$ $\left\{t: U_{t}(b)>U_{t}(a)\right\}$ and, consequently, $\sum_{t: U_{t}(b)>U_{t}(a)} w\left(U_{t}\right) \geq \sum_{t: U_{t}(c)>U_{t}(a)} w\left(U_{t}\right)$. Therefore, for the compatible probability distribution for which $a \succsim_{L}^{P} b$ we have,

$$
\sum_{t: U_{t}(a)>U_{t}(c)} w\left(U_{t}\right) \geq \sum_{t: U_{t}(a)>U_{t}(b)} w\left(U_{t}\right) \geq \sum_{t: U_{t}(b)>U_{t}(a)} w\left(U_{t}\right) \geq \sum_{t: U_{t}(c)>U_{t}(a)} w\left(U_{t}\right)
$$

and, consequently, for this compatible probability distribution $\sum_{t: U_{t}(a)>U_{t}(c)} w\left(U_{t}\right) \geq \sum_{t: U_{t}(c)>U_{t}(a)} w\left(U_{t}\right)$ being the thesis. 